Proyecciones Journal of Mathematics

Vol. 35, No 2, pp. 187-195, June 2016.

Universidad Católica del Norte

Antofagasta - Chile

\title{
Matrix transformation on statistically convergent sequence spaces of interval number sequences
}

\author{
Shyamal Debnath \\ Tripura University, India \\ and \\ Subrata Saha \\ Tripura University, India \\ Received : September 2015. Accepted : April 2016
}

\begin{abstract}
The main purpose of this paper is to introduce the necessary and sufficient conditions for the matrix of interval numbers $\bar{A}=\left(\bar{a}_{n k}\right)$ such that $\bar{A}$-transform of $\bar{x}=\left(\bar{x}_{k}\right)$ belongs to the sets $c_{0}^{S(i)} \cap l_{\infty}^{i}, c^{S(i)} \cap l_{\infty}^{i}$, where in particular $\bar{x} \in c_{0}^{S(i)} \cap l_{\infty}^{i}$ and $\bar{x} \in c^{S(i)} \cap l_{\infty}^{i}$ respectively.
\end{abstract}

Subjclass [2000] : 11B39, 40A35, 46B45.

Keywords : Interval number, sequence space, statistical convergence, matrix transformations. 


\section{Introduction}

The idea of statistical convergence was given by Zygmund [10] in the first edition of his monograph published in Warsaw in 1935. The concept of statistical convergence was introduced by Steinhaus [17] and Fast [15] and then reintroduced by Schoenberg [19] independently. Over the years and under different names, statistical convergence has been discussed in the theory of fourier analysis, ergodic theory, number theory, measure theory, trigonometric series, turnpike theory and banach spaces. Later on, statistical convergence turned out to be one of the most active areas of research in summability theory after the works of Fridy [20] and S̆alát [32]. For some very interesting investigations concerning statistical convergence, one may consult the papers of Cakalli [14], Miller [16], Maddox [18] and many others, where more references on this important summability method can be found.

Recently the sequence of interval numbers and usual convergence of sequences of interval numbers are studied by Chiao [21]. Later on, Şengönül and Eryılmaz [21] introduced and studied bounded and convergent sequence spaces of interval numbers and showed that these spaces are complete metric space. In the recent days, Esi [1-4] introduced and studied strongly

almost $\lambda$ - convergence and statistically almost $\lambda$ - convergence of interval numbers and lacunary sequence spaces of interval numbers respectively. For more information about interval numbers one may refer to Debnath et al. [28, 29, 30], Dwyer [23, 24], Fischer [25], Moore [26], Moore and Yang [27], Esi [5-9].

The theory of matrix transformations is a wide field in summability; it deals with the characterizations of classes of matrix mappings between sequence spaces by giving necessary and sufficient conditions on the entries of the infinite matrices. Matrix transformations in sequence spaces have been studied by different authors like Nanda [31], Tripathy [11, 12] and many others.

\section{Preliminaries}

We denote the set of all real valued closed intervals by $R(I)$. Any elements of $R(I)$ is called interval number and denoted by $\bar{x}=\left[x_{l}, x_{r}\right]$. The absolute value (magnitude or interval norm) of an interval number is defined by $|\bar{x}|=\max \left\{\left|x_{l}\right|,\left|x_{r}\right|\right\}$.

For $x_{1}, x_{2}, \in \mathrm{R}(\mathrm{I})$, we have $\bar{x}_{1}=\bar{x}_{2} \Leftrightarrow x_{l 1}=x_{l 2}, x_{r 1}=x_{r 2}, \bar{x}_{1}+\bar{x}_{2}$ 
$=\left\{x \in R: x_{l 1}+x_{l 2} \leq x \leq x_{r 1}+x_{r 2}\right\}$, and if $\alpha \geq 0$, then $\alpha \bar{x}=\{x \in R$ : $\left.\alpha x_{l 1} \leq x \leq \alpha x_{r 1}\right\}$ and if $\alpha<0$, then $\alpha \bar{x}=\left\{x \in R: \alpha x_{r 1} \leq x \leq \alpha x_{l 1}\right\}$, $\bar{x}_{1} \cdot \bar{x}_{2}=\left\{x \in R: \min \left\{x_{l 1} . x_{l 2}, x_{l 1} . x_{r 2}, x_{r 1} . x_{l 2}, x_{r 1} . x_{r 2}\right\} \leq x \leq \max \right.$ $\left.\left\{x_{l 1} . x_{l 2}, x_{l 1} . x_{r 2}, x_{r 1} . x_{l 2}, x_{r 1} . x_{r 2}\right\}\right\}$.

The set of all interval numbers $R(I)$ is a complete metric space defined by

$$
\mathrm{d}\left(\bar{x}_{1}, \bar{x}_{2}\right)=\max \left\{\left|x_{l 1}-x_{l 2}\right|,\left|x_{r 1}-x_{r 2}\right|\right\}
$$

In the special case $\bar{x}_{1}=[a, a]$ and $\bar{x}_{2}=[b, b]$, we obtain usual metric on the $R$ with

$$
\mathrm{d}\left(\bar{x}_{1}, \bar{x}_{2}\right)=|a-b|
$$

Let us define transformation $f$ from $N$ to $R(I)$ by $\mathrm{k} \rightarrow \mathrm{f}(\mathrm{k})=\bar{x}, \bar{x}=$ $\left(\bar{x}_{k}\right)$. Then $\left(\bar{x}_{k}\right)$ is called sequence of interval numbers. The $\bar{x}_{k}$ is called $k^{t h}$ term of sequence $\left(\bar{x}_{k}\right) . w^{i}$ denotes the set of all interval numbers with real terms and $c_{0}^{i}, c^{i}$ and $l_{\infty}^{i}$ denote the set of all null, convergent and bounded intervals with real terms.

Definition 2.1. A sequence $\bar{x}=\left(\bar{x}_{k}\right)$ of interval numbers is said to be convergent to the interval number $\bar{x}_{0}$ if for each $\epsilon>0$ there exists a positive number $k_{0}$ such that $\mathrm{d}\left(\bar{x}_{k}, \bar{x}_{0}\right)<\epsilon$ for all $\mathrm{k} \geq k_{0}$ and we denote it by $\lim _{k}$ $\bar{x}_{k}=\bar{x}_{0}$. Thus $\lim _{k} \bar{x}_{k}=\bar{x}_{0} \Leftrightarrow \lim _{k} x_{l k}=x_{l 0}$ and $\lim _{k} x_{r k}=x_{r 0}$.

Let, $\bar{x}=\left(\bar{x}_{k}\right)$ be a sequence of interval numbers.

$$
\bar{x}_{k}=\left[\frac{1}{k}, 1+\frac{1}{k^{2}}\right]
$$

which converges to the interval number $[0,1]$.

Definition 2.2. A sequence $\bar{x}=\left(\bar{x}_{k}\right)$ of interval numbers is said to be statistically convergent to the interval number $\bar{x}_{0}$ if for every $\epsilon>0, \lim _{n} \frac{1}{n}$ $\left|\left\{k \leq n: d\left(\bar{x}_{k}, \bar{x}_{0}\right) \geq \epsilon\right\}\right|=0$, denote it by writing stat-lim $\bar{x}_{k}=\bar{x}_{0}$.

Let, $\bar{x}=\left(\bar{x}_{k}\right)$ be a sequence of interval numbers.

$$
\bar{x}_{k}= \begin{cases}{\left[\mathrm{k}, \frac{1}{k}\right],} & k=n^{2}, n \in N ; \\ {[0,0],} & \text { otherwise. }\end{cases}
$$


which converges statistically to $\theta=[0,0]$.

Throughout this paper $l_{\infty}^{i}, c^{S(i)}$ and $c_{0}^{S(i)}$ denote the set of bounded, statistically convergent and statistically null sequences of interval numbers with real terms. We denote $\bar{e}_{k}$ as the interval sequence whose $k^{\text {th }}$ term is $[1,1]$ and the other terms are $\theta=[0,0]$.

Let $\bar{X}, \bar{Y}$ be two sequence spaces and let $\bar{A}=\left(\bar{a}_{n k}\right)$ be an infinite matrix of interval numbers $\bar{a}_{n k}$, where $n, k \in N$. Then, the matrix $\bar{A}$ defines the $\bar{A}$ - transformation from $\bar{X}$ into $\bar{Y}$, if for every sequence $\bar{x}=\left(\bar{x}_{k}\right) \in \bar{X}$ the sequence $\bar{A} \bar{x}=(\bar{A} \bar{x})_{n}$, the $\bar{A}$ - transform of $\bar{x}$ exists and is in $\bar{Y}$; where $(\bar{A} \bar{x})_{n}=\sum_{k} \bar{a}_{n k} \bar{x}_{k}$. For simplicity in notation, here and in what follows, the summation without limits run from 0 to $\infty$. By $\bar{A} \in(\bar{X}: \bar{Y})$ we mean the characterizations of matrices from $\bar{X}$ to $\bar{Y}$ i.e., $\bar{A}: \bar{X} \rightarrow \bar{Y}$. A sequence $\bar{x}$ is said to be $\bar{A}$ - summable to $\bar{x}_{0}$ if $\bar{A} \bar{x}$ converges to $\bar{x}_{0}$ which is called as the $\bar{A}$ - limit of $\bar{x}$.

For the sequence space $\bar{X}$, the matrix domain $\bar{X}_{\bar{A}}$ of an infinite matrix of interval numbers $\bar{A}$ is defined as

$$
\bar{X}_{\bar{A}}=\left\{\bar{x}=\left(\bar{x}_{k}\right): \bar{A} \bar{x} \in \bar{X}\right\}
$$

\section{Main Results}

Theorem 3.1: $\bar{A}=\left(\bar{a}_{n k}\right) \in\left(l_{\infty}^{i}: l_{\infty}^{i}\right)$ if and only if $\sup _{n \in N} \sum_{k}\left|\bar{a}_{n k}\right|<\infty$.

Proof: We assume that $\sup _{n \in N} \sum_{k}\left|\bar{a}_{n k}\right|<\infty$ holds and let $\bar{x}=\left(\bar{x}_{k}\right) \in l_{\infty}^{i}$. We also let $\bar{M}=\sup _{k} \bar{x}_{k}$.

Now, $\sup _{n \in N}\left|(\bar{A} \bar{x})_{n}\right|=\sup _{n \in N}\left|\sum_{k} \bar{a}_{n k} \bar{x}_{k}\right| \leq \bar{M}\left(\sup _{n \in N} \sum_{k}\left|\bar{a}_{n k}\right|\right)<\infty$ which leads to $\bar{A} \bar{x} \in l_{\infty}^{i}$.

Conversely, suppose that $\bar{A}=\left(\bar{a}_{n k}\right) \in\left(l_{\infty}^{i}: l_{\infty}^{i}\right)$, i.e., we have $\left(a_{l n k}\right) \in$ $\left(l_{\infty}: l_{\infty}\right)$ and $\left(a_{r n k}\right) \in\left(l_{\infty}: l_{\infty}\right)$. Then it can be shown that $\sup _{n \in N} \sum_{k}\left|a_{l n k}\right|<\infty$ and $\sup _{n \in N} \sum_{k}\left|a_{r n k}\right|<\infty$ as [Basar, (2011)].

Now the following lemmas will be used to prove the following theorem.

Lemma 3.1: The space $c^{S(i)} \cap l_{\infty}^{i}$ is a closed subspace of $l_{\infty}^{i}$. 
Proof: Let $\bar{x}=\left(\bar{x}_{k}\right) \in c^{S(i)} \cap l_{\infty}^{i}$. So we have, $\left(x_{l k}\right) \in c^{S} \cap l_{\infty}$ and $\left(x_{r k}\right) \in$ $c^{S} \cap l_{\infty}$. It can be shown as in the proof of theorem 2.1 of [ S̆alát, (1950)], that $c^{S} \cap l_{\infty}$ is closed subspace of $l_{\infty}$, i.e., the space $c^{S(i)} \cap l_{\infty}^{i}$ is a closed subspace of $l_{\infty}^{i}$.

Lemma 3.2: $\sup _{n, k}\left|a_{l n k}\right|<\infty$ and $\sup _{n, k}\left|a_{r n k}\right|<\infty$. If $\sum_{k} a_{l n k}$ and $\sum_{k} a_{r n k}$ converge uniformly and $S_{l n}=\sum_{k} a_{l n k}$ and $S_{r n}=\sum_{k} a_{r n k}$ for $n \in N, a_{l k}=$ stat $-\lim _{n \rightarrow \infty} a_{l n k}$ and $a_{r k}=$ stat $-\lim _{n \rightarrow \infty} a_{r n k}$ exist for each $k \in N$ then stat $-\lim _{n \rightarrow \infty} S_{l n}$ and stat $-\lim _{n \rightarrow \infty} S_{r n}$ exist and equal to $\sum_{k} a_{l k}$ and $\sum_{k} a_{r k}$ respectively, i.e., stat $-\lim _{n \rightarrow \infty} \bar{S}_{n}=\sum_{k} \bar{a}_{k}$

Proof: Using the similar technique as in the proof of lemma 2 [Tripathy, (1997), p-449], it can be shown that stat- $\lim _{n \rightarrow \infty} S_{l n}$ and $s t a t-l i m_{n \rightarrow \infty} S_{r n}$ exist and equal to $\sum_{k} a_{l k}$ and $\sum_{k} a_{r k}$ respectively, i.e., stat $-l i m_{n \rightarrow \infty} \bar{S}_{n}=$ $\sum_{k} \bar{a}_{k}$

Lemma 3.3: If $\sum_{k}\left|\bar{a}_{n k}\right|<\infty$ for each $n \in N$ and $\sum_{k}\left|\bar{a}_{n k}\right| \rightarrow \theta$, as $n \rightarrow \infty$ then $\sum_{k}\left|\bar{a}_{n k}\right|$ is uniformly convergent in $n \in N$.

Proof: Since $\sum_{k}\left|\bar{a}_{n k}\right| \rightarrow \theta$, so we have $\sum a_{l n k}<\infty$ and $\sum a_{r n k}<\infty$ seperately for each $n \in N$. Then we can proof the lemma as [Basar, (2011)].

Theorem 3.2: $\bar{A}=\left(\bar{a}_{n k}\right) \in\left(c^{S(i)} \cap l_{\infty}^{i}, c^{S(i)} \cap l_{\infty}^{i}\right)$ if and only if

$$
\sup _{n} \sum_{k}\left|\bar{a}_{n k}\right|<\infty
$$

and

$$
\bar{a}_{k}=\text { stat }-\lim _{n \rightarrow \infty} \bar{a}_{n k}, \text { exists for each } k \in N
$$

Proof: The necessity of (i) follows from the inclusion relation $\left(c^{S(i)} \cap l_{\infty}^{i}, c^{S(i)} \cap l_{\infty}^{i}\right) \subset\left(c^{S(i)} \cap l_{\infty}^{i}, l_{\infty}^{i}\right)$. That of (ii) follows on considering the sequence $\left(\bar{e}_{k}\right)$. For the sufficiency, by lemma 3.1 and (ii), it is enough to show that $\sum_{k \geq m} \bar{a}_{n k} \bar{x}_{k} \rightarrow \theta, m \rightarrow \infty$ uniformly in $\mathrm{n}$.

Now,

$$
\sum_{k=m}^{\infty}\left|\bar{a}_{n k} \bar{x}_{k}\right| \leq \sup _{k} \bar{x}_{k} \sum_{k=1}^{\infty}\left|\bar{a}_{n k}\right|
$$

Let $\bar{M}=\sup _{k} \bar{x}_{k}$, since $\left(\bar{x}_{k}\right)$ is bounded. Then for every $\varepsilon>0$, by (i) there exists $m_{0}$ such that

$$
\sum_{k}\left|\bar{a}_{n k}\right|<\varepsilon \bar{M}^{-1}
$$

Thus $\sum_{k>m_{0}}^{\infty}\left|\bar{a}_{n k} \bar{x}_{k}\right|<\varepsilon$ implies $\sum_{k}\left|\bar{a}_{n k} \bar{x}_{k}\right| \rightarrow \theta$ as $m_{0} \rightarrow \infty$, uniformly in $\mathrm{n}$ as lemma 3.1. Hence, by lemma $3.2, \bar{A}_{n} \bar{x} \in c^{S(i)} \cap l_{\infty}^{i}$. 
Corollary 3.1 $\bar{A}=\left(\bar{a}_{n k}\right) \in\left(c^{S(i)} \cap l_{\infty}^{i}, c_{0}^{S(i)} \cap l_{\infty}^{i}\right)$ if and only if (i) and (ii) with $\bar{a}_{k}=\theta$ holds.

Theorem 3.3: $\bar{A}=\left(\bar{a}_{n k}\right) \in\left(c_{0}^{S(i)} \cap l_{\infty}^{i}, c^{S(i)} \cap l_{\infty}^{i}\right)$ if and only if

$$
\sup _{n} \sum_{k}\left|\bar{a}_{n k}\right|<\infty
$$

and

$$
\text { stat }-\lim _{n \rightarrow \infty} \bar{a}_{n k} \text { exists for each fixed } k \in N \text {. }
$$

Proof: The necessity of (i) follows from the inclusion relation $\left(c_{0}^{S(i)} \cap l_{\infty}^{i}, c^{S(i)} \cap l_{\infty}^{i}\right) \subset\left(c_{0}^{S(i)} \cap l_{\infty}^{i}, l_{\infty}^{i}\right)$. That of (ii) follows on considering the sequence $\left(\bar{e}_{k}\right)$.

Conversely, let $\bar{x}=\left(\bar{x}_{k}\right) \in c_{0}^{S(i)} \cap l_{\infty}^{i}$. As in theorem 3.1, $\sum_{k>m_{0}} \bar{a}_{n k} \bar{x}_{k}$ converges to $\theta=[0,0]$ uniformly in $\mathrm{n}$. Since, $\bar{a}_{n k} \in c^{S(i)} \cap l_{\infty}^{i}$, so $\sum_{k \leq m_{0}} \bar{a}_{n k} \bar{x}_{k} \in c^{S(i)} \cap l_{\infty}^{i}$. Hence by lemma 3.2 we have $\bar{A}_{n} \bar{x} \in c^{S(i)} \cap l_{\infty}^{i}$. This completes the proof.

Corollary 3.2 $\bar{A}=\left(\bar{a}_{n k}\right) \in\left(c_{0}^{S(i)} \cap l_{\infty}^{i}, c_{0}^{S(i)} \cap l_{\infty}^{i}\right)$ if and only if $\sup _{n} \sum_{k}\left|\bar{a}_{n k}\right|<\infty$ and stat $-\lim _{n \rightarrow \infty} \bar{a}_{n k}=\theta$, where $\theta=[0,0]$ for each fixed $k \in N$.

\section{References}

[1] A. Esi, Strongly almost $\lambda$ - convergence and statistically almost $\lambda$ convergence of interval numbers, Scientia Magna 7 (2): pp. 117-122, (2011).

[2] A. Esi, A new class of interval numbers, J. Qafqaz Univ. 31: pp. 98-102, (2011).

[3] A. Esi, Lacunary sequence spaces of interval numbers, Thai J. Math., 10(2): pp. 445-451, (2012).

[4] A. Esi, $\lambda$ - Sequence spaces of interval numbers, Appl. Math. and Inform. Sci., 8(3): pp. 1099-1102, (2014). 
[5] A. Esi, Double lacunary sequence spaces of double sequence of interval numbers, Proyecciones, 31(1): pp. 297-306, (2012).

[6] A. Esi, Statistical and lacunary statistical convergence of interval numbers in topological groups, Acta Scientarium. Techno., 36(3): pp. 491495, (2014).

[7] A. Esi and N. Braha, On asymptotically -statistical equivalent sequences of interval numbers, Acta Scientarium. Techno., 35(3): pp. 515-520, (2013).

[8] A. Esi and A. Esi, Asymptotically lacunary statistically equivalent sequences of interval numbers, Int. J. Math. Appl., 1(1): pp. 43-48, (2013).

[9] A. Esi and B. Hazarika, Some ideal convergence of double -interval number sequences defined by Orlicz function, Global J. Math. Anal.,1(3): pp. 110-116, (2013).

[10] A. Zygmund, Trigonometric Series, Cambridge University Press, Cambridge, UK. (1979).

[11] B.C. Tripathy, Matrix transformation between some classes of sequences, J. Math. Anal. Appl., 206: pp. 448-450, (1997).

[12] B.C. Tripathy, Matrix transformation between series and sequences, Bull. Malaysian Math. Soc. (Second series), 21: pp. 17-20, (1998).

[13] F. Basar, Summability theory and its applications, Bentham Science, (2011).

[14] H. Cakalli, A study on statistical convergence, Funct. Anal. Approx. Comput., 1(2): pp. 19-24, (2009).

[15] H. Fast, Sur la convergence statistique, Colloq. Math. 2: pp. 241-244, (1951).

[16] H. I. Miller, A measure theoretical subsequence characterization of statical convergence, Trans. Am. Math. Soc., 347(5): pp. 1811-1819, (1995).

[17] H. Steinhus,Sur la convergence ordinaire et la convergence asymptotique, Colloq. Math. 2: pp. 73-74, (1951). 
[18] I.J. Maddox, On strong almost cconvergence, Math. Proc. Camb. Philos. Soc., 85(2): pp. 345-350, (1979).

[19] I. J. Schoenberg, The integrability of certain functions and related summability methods, Am. Math. Mon., 66(5): pp. 361-375, (1959).

[20] J. A. Fridy, On statistical convergence, Analysis, 5(4): pp. 301-313, (1985).

[21] Kuo-Ping Chiao, Fundamental properties of interval vector max-norm, Tamsui Oxford J. Math. Sci., 18(2): pp. 219-233, (2002).

[22] M. Şengönül and A.Eryılmaz, On the sequence spaces of interval numbers, Thai J. Math, 8(3): pp. 503-510, (2010).

[23] P. S. Dwyer, Linear Computation, New York, Wiley, (1951).

[24] P.S. Dwyer, Erros of matrix computation, simultaneous equations and eigenvalues, National Bureu of Standarts, Applied Mathematics Series, 29: pp. 49-58, (1953).

[25] P. S. Fischer, Automatic propagated and round-off error analysis, Paper presented at the $13^{\text {th }}$ national meeting of the Association for Computing Machinary, (1958).

[26] R. E. Moore, Automatic error analysis in digital computation, LSMD48421,Lockheed Missiles and Space Company, (1959).

[27] R. E. Moore and C. T. Yang, Theory of an interval algebra and its application to numeric analysis, RAAG Memories II, Gaukutsu Bunken Fukeyu-kai, Tokyo, (1958).

[28] S. Debnath, A. J. Dutta and S. Saha, Regular matrix of interval numbers based on fibonacci numbers, Afr. Mat., 26(7): pp. 1379-1385, (2015).

[29] S. Debnath, B. Sarma and S. Saha, On some sequence spaces of interval vectors, Afr. Mat., 26(5): pp. 673-678, (2015).

[30] S. Debnath and S. Saha, On Statistically Convergent Sequence Spaces of Interval Numbers, Proceedings of IMBIC, 3: pp. 178-183, (2014).

[31] S. Nanda, Matrix transformations between sequence spaces, Queen's papers in pure and Appl. Math., pp. 74, (1986). 
Matrix transformation on statistically convergent sequence spaces...195

[32] T. Salát, On statistical convergence sequences of real numbers, Math. Slovaca, 30: pp. 139-150, (1950).

\author{
Shyamal Debnath \\ Department of Mathematics \\ Tripura University (A Central University) \\ Suryamaninagar - 799022, \\ Agartala, \\ India \\ e-mail : shyamalnitamath@gmail.com \\ and

\section{Subrata Saha} \\ Department of Mathematics \\ Tripura University (A Central University) \\ Suryamaninagar - 799022, \\ Agartala, \\ India \\ e-mail : subratasaha2015@gmail.com
}

